PROCEEDINGS OF THE

AMERICAN MATHEMATICAL SOCIETY

Volume 125, Number 12, December 1997, Pages 3641-3645

S $0002-9939(97) 04033-1$

\title{
STRONG CONVERGENCE OF APPROXIMATED SEQUENCES FOR NONEXPANSIVE MAPPINGS IN BANACH SPACES
}

\author{
NAOKI SHIOJI AND WATARU TAKAHASHI \\ (Communicated by Palle E. T. Jorgensen)
}

\begin{abstract}
In this paper, we study the convergence of the sequence defined by $x_{0} \in C, x_{n+1}=\alpha_{n} x+\left(1-\alpha_{n}\right) T x_{n}, \quad n=0,1,2, \ldots$,

where $0 \leq \alpha_{n} \leq 1$ and $T$ is a nonexpansive mapping from a closed convex subset of a Banach space into itself.
\end{abstract}

\section{INTRODUCTION}

Let $C$ be a closed, convex subset of a Banach space $E$ and let $T$ be a nonexpansive mapping from $C$ into $C$, i.e., $\|T x-T y\| \leq\|x-y\|$ for all $x, y \in C$. We deal with the iterative process

$$
x_{0} \in C, x_{n+1}=\alpha_{n} x+\left(1-\alpha_{n}\right) T x_{n}, \quad n=0,1,2, \ldots,
$$

where $0 \leq \alpha_{n} \leq 1$ and $\alpha_{n} \rightarrow 0$. Concerning this process, Reich [5] posed the following problem:

Problem. Let $E$ be a Banach space. Is there a sequence $\left\{\alpha_{n}\right\}$ such that whenever a weakly compact, convex subset $C$ of $E$ possesses the fixed point property for nonexpansive mappings, then the sequence $\left\{x_{n}\right\}$ defined by (1.1) converges to a fixed point of $T$ for all $x$ in $C$ and all nonexpansive $T: C \rightarrow C$ ?

Though Reich $[4,5]$ showed an answer in the case when $E$ is uniformly smooth and $\alpha_{n}=n^{-a}$ with $0<a<1$, the problem has been generally open. Recently, Wittmann [7] solved the problem in the case when $E$ is a Hilbert space and $\left\{\alpha_{n}\right\}$ satisfies

$$
0 \leq \alpha_{n} \leq 1, \lim _{n \rightarrow \infty} \alpha_{n}=0, \sum_{n=0}^{\infty} \alpha_{n}=\infty \text { and } \sum_{n=0}^{\infty}\left|\alpha_{n+1}-\alpha_{n}\right|<\infty .
$$

In this paper, we extend Wittmann's result to Banach spaces. Our result is the following:

Theorem. Let $E$ be a Banach space whose norm is uniformly Gâteaux differentiable and let $C$ be a closed, convex subset of $E$. Let $T$ be a nonexpansive mapping from $C$ into $C$ such that the set $F(T)$ of fixed points of $T$ is nonempty. Let $\left\{\alpha_{n}\right\}$

Received by the editors February 6, 1996 and, in revised form, July 15, 1996.

1991 Mathematics Subject Classification. Primary 47H09, 49M05.

Key words and phrases. Nonexpansive mappings, strong convergence, Banach limits, iteration.

(C)1997 American Mathematical Society 
be a sequence which satisfies (1.2). Let $x \in C$ and let $\left\{x_{n}\right\}$ be the sequence defined by (1.1). Assume that $\left\{z_{t}\right\}$ converges strongly to $z \in F(T)$ as $t \downarrow 0$, where for $0<t<1, z_{t}$ is a unique element of $C$ which satisfies $z_{t}=t x+(1-t) T z_{t}$. Then $\left\{x_{n}\right\}$ converges strongly to $z$.

If $C$ satisfies additional assumptions then $\left\{z_{t}\right\}$ defined above converges strongly to a fixed point of $T$. We know the following $[4,6]$ :

Let $E$ be a Banach space whose norm is uniformly Gâteaux differentiable, let $C$ be a weakly compact, convex subset of $E$ and let $T$ be a nonexpansive mapping from $C$ into $C$. Let $x \in C$ and let $z_{t}$ be a unique element of $C$ which satisfies $z_{t}=t x+(1-t) T z_{t}$ for $0<t<1$. Assume that each nonempty, $T$-invariant, closed, convex subset of $C$ contains a fixed point of $T$. Then $\left\{z_{t}\right\}$ converges strongly to a fixed point of $T$.

So our theorem gives an answer to Reich's problem in the case when the norm of $E$ is uniformly Gâteaux differentiable and each nonempty, closed, convex subset of $C$ possesses the fixed point property for nonexpansive mappings.

\section{Preliminaries and notations}

Throughout this paper, all vector spaces are real and we denote by $\mathbb{N}$ and $\mathbb{N}_{+}$, the set of all nonnegative integers and the set of all positive integers, respectively. Let $E$ be a Banach space and let $E^{\prime}$ be its dual. The value of $y \in E^{\prime}$ at $x \in E$ will be denoted by $\langle x, y\rangle$. We also denote by $J$ the duality mapping from $E$ into $2^{E^{\prime}}$, i.e.,

$$
J x=\left\{y \in E^{\prime}:\langle x, y\rangle=\|x\|^{2}=\|y\|^{2}\right\}, \quad x \in E .
$$

Let $U=\{x \in E:\|x\|=1\}$. The norm of $E$ is said to be uniformly Gâteaux differentiable if, for each $y \in U$, the limit

$$
\lim _{t \rightarrow 0} \frac{\|x+t y\|-\|x\|}{t}
$$

exists uniformly for $x \in U . E$ is said to be uniformly smooth if the limit (2.1) exists uniformly for $x, y \in U$. It is well known that if the norm of $E$ is uniformly Gâteaux differentiable then the duality mapping is single-valued and norm to weak star, uniformly continuous on each bounded subset of $E$.

Let $\mu$ be a continuous, linear functional on $l^{\infty}$ and let $\left(a_{0}, a_{1}, \cdots\right) \in l^{\infty}$. We write $\mu_{n}\left(a_{n}\right)$ instead of $\mu\left(\left(a_{0}, a_{1}, \cdots\right)\right)$. We call $\mu$ a Banach limit [1] when $\mu$ satisfies $\|\mu\|=\mu_{n}(1)=1$ and $\mu_{n}\left(a_{n+1}\right)=\mu_{n}\left(a_{n}\right)$ for all $\left(a_{0}, a_{1}, \cdots\right) \in l^{\infty}$.

To prove our result, we need the following propositions, which can be deduced by the same lines as those in [3]. For the sake of completeness, we give the proofs in our appendix.

Proposition 1. Let a be a real number and let $\left(a_{0}, a_{1}, \cdots\right) \in l^{\infty}$. Then $\mu_{n}\left(a_{n}\right) \leq a$ for all Banach limits $\mu$ if and only if for each $\varepsilon>0$, there exists $p_{0} \in \mathbb{N}_{+}$such that

$$
\frac{a_{n}+a_{n+1}+\cdots+a_{n+p-1}}{p}<a+\varepsilon \quad \text { for all } p \geq p_{0} \text { and } n \in \mathbb{N} .
$$

Proposition 2. Let a be a real number and let $\left(a_{0}, a_{1}, \cdots\right) \in l^{\infty}$ such that $\mu_{n}\left(a_{n}\right) \leq$ a for all Banach limits $\mu$ and $\varlimsup_{n \rightarrow \infty}\left(a_{n+1}-a_{n}\right) \leq 0$. Then $\varlimsup_{n \rightarrow \infty} a_{n} \leq a$. 


\section{Proof of Theorem}

The following is obtained in [7]. For the sake of completeness, we give the proof.

Lemma 1. $\lim _{n \rightarrow \infty}\left\|x_{n+1}-x_{n}\right\|=0$.

Proof. We remark that $\left\{x_{n}\right\}$ and $\left\{T x_{n}\right\}$ are bounded by $F(T) \neq \emptyset$. Set $M=$ $\sup \left\{\left\|T x_{n}\right\|: n \in \mathbb{N}\right\}$. Then since $\left\|x_{n+1}-x_{n}\right\| \leq\left|\alpha_{n}-\alpha_{n-1}\right|(\|x\|+M)+$ $\left(1-\alpha_{n}\right)\left\|x_{n}-x_{n-1}\right\|$ for each $n \in \mathbb{N}_{+}$, we have

$$
\begin{aligned}
& \left\|x_{n+m+1}-x_{n+m}\right\| \\
& \quad \leq\left(\sum_{k=m}^{n+m-1}\left|\alpha_{k+1}-\alpha_{k}\right|\right)(\|x\|+M)+\left(\prod_{k=m}^{n+m-1}\left(1-\alpha_{k+1}\right)\right)\left\|x_{m+1}-x_{m}\right\| \\
& \quad \leq\left(\sum_{k=m}^{n+m-1}\left|\alpha_{k+1}-\alpha_{k}\right|\right)(\|x\|+M)+\exp \left(-\sum_{k=m}^{n+m-1} \alpha_{k+1}\right)\left\|x_{m+1}-x_{m}\right\|
\end{aligned}
$$

for all $m, n \in \mathbb{N}$. So the boundedness of $\left\{x_{n}\right\}$ and $\sum_{k=0}^{\infty} \alpha_{k}=\infty$ yield

$$
\varlimsup_{n \rightarrow \infty}\left\|x_{n+1}-x_{n}\right\|=\varlimsup_{n \rightarrow \infty}\left\|x_{n+m+1}-x_{n+m}\right\| \leq\left(\sum_{k=m}^{\infty}\left|\alpha_{k+1}-\alpha_{k}\right|\right)(\|x\|+M)
$$

for all $m \in \mathbb{N}$. Hence by $\sum_{k=0}^{\infty}\left|\alpha_{k+1}-\alpha_{k}\right|<\infty$, we get the conclusion.

Using Proposition 2, we obtain the following.

Lemma 2. $\varlimsup_{n \rightarrow \infty}\left\langle x-z, J\left(x_{n}-z\right)\right\rangle \leq 0$.

Proof. Let $\mu$ be a Banach limit and let $0<t<1$. Since $\left\{\alpha_{n}\right\}$ converges to $0, T$ is nonexpansive and $\mu$ is a Banach limit, we get

$$
\mu_{n}\left\|x_{n}-T z_{t}\right\|^{2} \leq \mu_{n}\left\|x_{n}-z_{t}\right\|^{2} .
$$

From $(1-t)\left(x_{n}-T z_{t}\right)=\left(x_{n}-z_{t}\right)-t\left(x_{n}-x\right)$, we have

$$
\begin{aligned}
(1-t)^{2}\left\|x_{n}-T z_{t}\right\|^{2} & \geq\left\|x_{n}-z_{t}\right\|^{2}-2 t\left\langle x_{n}-x, J\left(x_{n}-z_{t}\right)\right\rangle \\
& =(1-2 t)\left\|x_{n}-z_{t}\right\|^{2}+2 t\left\langle x-z_{t}, J\left(x_{n}-z_{t}\right)\right\rangle
\end{aligned}
$$

for each $n \in \mathbb{N}$. These inequalities yield

$$
\frac{t}{2} \mu_{n}\left\|x_{n}-z_{t}\right\|^{2} \geq \mu_{n}\left\langle x-z_{t}, J\left(x_{n}-z_{t}\right)\right\rangle .
$$

Tending $t$ to 0 , we get

$$
0 \geq \mu_{n}\left\langle x-z, J\left(x_{n}-z\right)\right\rangle,
$$

because $E$ has a uniformly Gâteaux differentiable norm. On the other hand, we have

$$
\lim _{n \rightarrow \infty}\left|\left\langle x-z, J\left(x_{n+1}-z\right)\right\rangle-\left\langle x-z, J\left(x_{n}-z\right)\right\rangle\right|=0
$$

by Lemma 1 . Hence by Proposition 2, we obtain

$$
\varlimsup_{n \rightarrow \infty}\left\langle x-z, J\left(x_{n}-z\right)\right\rangle \leq 0 .
$$


Now we can prove our theorem.

Proof of Theorem. Since $\left(1-\alpha_{n}\right)\left(T x_{n}-z\right)=\left(x_{n+1}-z\right)-\alpha_{n}(x-z)$, we have

$$
\left\|\left(1-\alpha_{n}\right)\left(T x_{n}-z\right)\right\|^{2} \geq\left\|x_{n+1}-z\right\|^{2}-2 \alpha_{n}\left\langle x-z, J\left(x_{n+1}-z\right)\right\rangle,
$$

which yields

$$
\left\|x_{n+1}-z\right\|^{2} \leq\left(1-\alpha_{n}\right)\left\|x_{n}-z\right\|^{2}+2\left(1-\left(1-\alpha_{n}\right)\right)\left\langle x-z, J\left(x_{n+1}-z\right)\right\rangle
$$

for each $n \in \mathbb{N}$. Let $\varepsilon>0$. By Lemma 2 , there exists $m \in \mathbb{N}$ such that

$$
\left\langle x-z, J\left(x_{n}-z\right)\right\rangle \leq \frac{\varepsilon}{2}
$$

for all $n \geq m$. Then we have

$$
\left\|x_{n+m}-z\right\|^{2} \leq\left(\prod_{k=m}^{n+m-1}\left(1-\alpha_{k}\right)\right)\left\|x_{m}-z\right\|^{2}+\left(1-\prod_{k=m}^{n+m-1}\left(1-\alpha_{k}\right)\right) \varepsilon
$$

for all $n \in \mathbb{N}$. Hence by $\sum_{k=0}^{\infty} \alpha_{k}=\infty$, we get

$$
\varlimsup_{n \rightarrow \infty}\left\|x_{n}-z\right\|^{2}=\varlimsup_{n \rightarrow \infty}\left\|x_{n+m}-z\right\|^{2} \leq \varepsilon .
$$

Since $\varepsilon$ is an arbitrary positive real number, $\left\{x_{n}\right\}$ converges strongly to $z$.

Remark. Halpern [2] showed that $\alpha_{n} \rightarrow 0$ and $\sum_{n=0}^{\infty} \alpha_{n}=\infty$ are necessary conditions for the convergence of the sequence $\left\{x_{n}\right\}$ defined by (1.1). The condition $\sum_{n=0}^{\infty}\left|\alpha_{n+1}-\alpha_{n}\right|<\infty$ is used only to show $x_{n+1}-x_{n} \rightarrow 0$. For other conditions which ensure $x_{n+1}-x_{n} \rightarrow 0$, see [7].

\section{APPENDIX}

In this appendix, we prove Proposition 1 and Proposition 2.

Proof of Proposition 1. First we shall prove the only if part. Assume that $\mu_{n}\left(a_{n}\right) \leq$ $a$ for all Banach limits $\mu$. Define a sublinear functional $q$ from $l^{\infty}$ into the set of real numbers by

$$
q\left(\left(b_{0}, b_{1}, \cdots\right)\right)=\varlimsup_{p \rightarrow \infty} \sup _{n \in \mathbb{N}} \frac{1}{p} \sum_{i=n}^{n+p-1} b_{i}, \quad\left(b_{0}, b_{1}, \cdots\right) \in l^{\infty}
$$

We write $q_{n}\left(b_{n}\right)$ instead of $q\left(\left(b_{0}, b_{1}, \cdots\right)\right)$ for $\left(b_{0}, b_{1}, \cdots\right) \in l^{\infty}$. By the Hahn-Banach theorem, there exists a linear functional $\mu$ from $l^{\infty}$ into the set of real numbers such that $\mu \leq q$ and $\mu_{n}\left(a_{n}\right)=q_{n}\left(a_{n}\right)$. It is easy to see that $\mu$ is a Banach limit. From the assumption, we have $q_{n}\left(a_{n}\right) \leq a$. So for each $\varepsilon>0$, there exists $p_{0} \in \mathbb{N}_{+}$which satisfies (2.2).

Next we shall prove the if part. Assume that for each $\varepsilon>0$, there exists $p_{0} \in \mathbb{N}_{+}$ which satisfies (2.2). Let $\mu$ be a Banach limit and let $\varepsilon>0$. By the hypothesis, there exists $p_{0} \in \mathbb{N}_{+}$which satisfies (2.2). So we have

$$
\mu_{n}\left(a_{n}\right)=\mu_{n}\left(\frac{a_{n}+a_{n+1}+\cdots+a_{n+p_{0}-1}}{p_{0}}\right) \leq a+\varepsilon .
$$

Since $\varepsilon$ is an arbitrary positive real number, we get $\mu_{n}\left(a_{n}\right) \leq a$. 
Proof of Proposition 2. Let $\varepsilon>0$. By Proposition 1, there exists $p \geq 2$ such that

$$
\frac{a_{n}+a_{n+1}+\cdots+a_{n+p-1}}{p}<a+\frac{\varepsilon}{2}
$$

for all $n \in \mathbb{N}$. Choose $n_{0} \in \mathbb{N}$ such that $a_{n+1}-a_{n}<\varepsilon /(p-1)$ for all $n \geq n_{0}$. Let $n \geq n_{0}+p$. Then we have

$$
\begin{aligned}
a_{n}= & a_{n-i}+\left(a_{n-i+1}-a_{n-i}\right)+\left(a_{n-i+2}-a_{n-i+1}\right)+\cdots+\left(a_{n}-a_{n-1}\right) \\
& \leq a_{n-i}+\frac{i \varepsilon}{p-1}
\end{aligned}
$$

for each $i=0,1, \cdots, p-1$. So we get

$$
a_{n} \leq \frac{a_{n}+a_{n-1}+\cdots+a_{n-p+1}}{p}+\frac{1}{p} \cdot \frac{p(p-1)}{2} \cdot \frac{\varepsilon}{p-1} \leq a+\varepsilon .
$$

Hence we have

$$
\varlimsup_{n \rightarrow \infty} a_{n} \leq a+\varepsilon .
$$

Since $\varepsilon$ is an arbitrary positive real number, we get the conclusion.

\section{ACKNOWLEDGMENT}

The authors wish to express their sincere thanks to the referee for giving a simple proof of Proposition 1.

\section{REFERENCES}

[1] S. Banach, Théorie des opérations linéaires, Monografie Mat., PWN, Warszawa, 1932.

[2] B. Halpern, Fixed points of nonexpanding maps, Bull. Amer. Math. Soc. 73 (1967), 957-961. MR 36:2022

[3] G. G. Lorentz, A contribution to the theory of divergent series, Acta Math. 80 (1948), 167190. MR 10:367e

[4] S. Reich, Strong convergence theorems for resolvents of accretive operators in Banach spaces, J. Math. Anal. Appl. 75 (1980), 287-292. MR 82a:47050

[5] S. Reich, Some problems and results in fixed point theory, Contemp. Math. 21 (1983), 179-187. MR 85e:47082

[6] W. Takahashi and Y. Ueda, On Reich's strong convergence theorems for resolvents of accretive operators, J. Math. Anal. Appl. 104 (1984), 546-553. MR 86c:47070

[7] R. Wittmann, Approximation of fixed points of nonexpansive mappings, Arch. Math. 58 (1992), 486-491. MR 93c:47069

Faculty of Engineering, Tamagawa University, Tamagawa-Gakuen, Machida, Tokyo 194, JAPAN

E-mail address: shioji@eng.tamagawa.ac.jp

Department of Information Science, Tokyo Institute of Technology, Oh-okayama, Meguro-Ku, Tokyo 152, JAPAN

E-mail address: wataru@is.titech.ac.jp 